\title{
STABLE MAPS AND SCHWARTZ MAPS
}

\author{
BY \\ ANDRE DE KORVIN
}

1. Introduction. In the present paper $M$ and $N$ will denote two von Neumann algebras where $N \subset M$. If $\mathscr{A}$ is any von Neumann algebra, $\mathscr{A}^{\prime}$ will denote the commutant of $\mathscr{A} . N^{c}$ will denote the relative commutant of $N$ in $M$, i.e. $N^{c}=$ $N^{\prime} \cap M . U(N)$ will denote all unitary operators of $N$. Let $G$ be a group of unitaries of $M$. Let $\phi$ be a linear map of $M$ into $M . \phi$ is called $G$-stable if $\phi\left(U X U^{-1}\right)=\phi(X)$ for all $X$ in $M$ and all $U$ in $G . S(G, M)$ will denote all Schwartz maps which are $G$-stable. The purpose of this paper is to study the existence and properties of $G$ stable expectations. The main results contained here are:

THEOREM 1. Let $\operatorname{Tr}$ be a faithful, semifinite trace on $M$. Let $L$ be a von Neumann subalgebra of $M$ such that $\operatorname{Tr}$ restricted to $L$ is semifinite. Then there exists a normal, faithful, $U\left(L^{c}\right)$ stable expectation $\phi$ of $M$ on $L$ such that $\operatorname{Tr}(A \phi(X))=\operatorname{Tr}(A X)$ for all $X$ in $M$ and all $A$ in $L$ for which $\operatorname{Tr}|A|<\infty$.

THEOREM 2. Suppose $M$ has a faithful, normal, semifinite trace, call it $\operatorname{Tr}$. Suppose $S(G, M)$ is sufficiently large, then there exists a faithful, normal, $U\left(N^{c c}\right)$ stable expectation of $M$ on $N^{c}$.

As corollary to the above theorem, it follows that with the hypothesis of Theorem 2, $N$ is finite, $N^{c}$ can not be purely infinite. Moreover if $M$ is of type I so is $N^{c}$. Another corollary to Theorem 2 is that if $S(G, L(h))$ has sufficiently many maps, then the von Neumann algebra $N$ generated by $G$ is atomic.

Next a notion of equivalence of two unitary groups will be defined. Two groups of unitary operators are equivalent if they generate the same von Neumann algebra.

Theorem 3. Assume $S(G, L(h))$ contains a normal map, then $G$ is equivalent to a countable direct sum of finite groups.

The next result is a sort of converse to Theorem 3 .

THEOREM 4. If $G$ has the property $(F)$, then $G$ is a countable direct sum of finite groups and $S(G, M)$ has sufficiently many maps.

A corollary to Theorem 3 is that if $N$ is a finite atomic von Neumann algebra, then $N$ is generated by a direct sum of finite unitary groups.

Received by the editors October 30, 1968 and, in revised form, August 25, 1969.

Copyright (C) 1970, American Mathematical Society 
Next uniqueness of expectations of certain type will be considered. The main result of this section is:

THEOREM 5. Assume that

(1) $N^{c} \subset N$,

(2) $N$ is finite,

(3) $M$ is semifinite.

Then there exists at most one normal expectation $\phi$ of $M$ on $N$.

2. Preliminaries.

Definition. Let $\phi$ be a map of $M$ into $N$ which preserves the identity. Assume that $\phi$ is a positive linear map and that $\phi(A X)=A \phi(X)$ for all $A$ in $N$ and $X$ in $M$. $\phi$ will then be called an expectation of $M$ in $N$.

It is trivial to see that $\phi$ is onto $N$ and that $\phi$ is a bounded map. The motion of expectations in von Neumann algebras was studied in [2], [7], and [9].

Definition. Let $\phi$ be an expectation of $M$ on $N, \phi$ is called normal if $\phi\left(\operatorname{Sup} A_{\alpha}\right)$ $=\operatorname{Sup} \phi\left(A_{\alpha}\right)$ for any increasing net of uniformly bounded selfadjoint operators.

$\phi$ is called faithful if, given a positive operator $A$ such that $\phi(A)=0$, then $A=0$.

Let $\phi_{\alpha}$ be a set of expectations of $M$ onto $N$. The set $\phi_{\alpha}$ is called complete if given a positive operator $A$ such that $\phi_{\alpha}(A)=0$ then $A=0$.

Definition. Let $G$ be a subgroup of $U(M)$. By a Schwartz map relative to $(G, M)$ one means a linear map of $M$ into itself such that

(1) $P(X)=U P(X) U^{-1}$ for all $U$ in $G$ and all $X$ in $M$,

(2) $P(X)$ is in $C_{G}[X]$ where $C_{G}[X]$ denotes the weak closure of the convex hull generated by elements of the type $U X U^{-1}$ as $U$ ranges over $G$.

For more information on Schwartz maps see [6].

$S(G, M)$ will denote all Schwartz maps relative to $(G, M)$ which are $G$-stable, i.e. $P(X)=P\left(V X V^{-1}\right)$ for all $V$ in $G . S(G, M)$ will be called sufficient if for any positive operator $X$ in $M$ such that $P(X)=0$ for all $P$ in $S(G, M)$ then $X=0$.

Definition. A group $G$ is said to be amenable as a discrete group if there exists a finitely additive probability measure $\mu$ on the field of all subsets of $G$ such that $\mu(x E)=\mu(E)$. For more information on amenable groups see [4] and [5].

\section{Stable maps and Schwartz maps.}

LEMMA 1. If there exists a complete set of $U$-stable expectations of $M$ on $N$ then $U$ is in $N^{c}$.

Proof. Let $V$ be a unitary of $N$. Let $\phi_{\alpha}$ be a complete set of $U$-stable expectations, then $\phi_{\alpha}\left(U V U^{-1} V^{-1}\right)=\phi_{\alpha}\left(V U^{-1} V^{-1} U\right)=V \phi_{\alpha}\left(U^{-1} V^{-1} U\right)$. This by stability. Also $V \phi_{\alpha}\left(V^{-1}\right)=V V^{-1}=I$. Let $W=U V U^{-1} V^{-1}$. Then $\phi_{\alpha}\left[(W-I)^{*}(W-I)\right]=0$. By completeness $W=I$ or $U V=V U$. So $U$ is in $N^{c}$.

Lemma 2. A normal $U(N)$ stable expectation of $M$ on $N^{c}$ is faithful. 
Proof. Let $\phi$ be the expectation. Let $I=\left\{A \mid A \in M, \phi\left(A^{*} A\right)=0\right\}$ clearly as $\phi\left[(X A)^{*}(X A)\right] \leqq\|X\|^{2} \phi\left(A^{*} A\right)=0$ and $(A+B)^{*}(A+B) \leqq 2\left(A^{*} A+B^{*} B\right) . I$ is a left ideal.

Now to show that $I$ is ultra-weakly closed. The ultra-weak closure of $I$ coincides with its ultra-strong closure. Let $X_{\alpha}$ be a net in $I$ converging ultra-strongly to $X$, then $\left(X_{\alpha}-X\right)^{*}\left(X_{\alpha}-X\right)$ converges ultra-weakly to 0 . Hence $\phi\left[\left(X_{\alpha}-X\right)^{*}\left(X_{\alpha}-X\right)\right]$ converges to 0 ultra-weakly (normality). As

$$
\phi\left(X_{\alpha}-X\right)^{*} \phi\left(X_{\alpha}-X\right) \leqq \phi\left(X_{\alpha}-X\right)^{*}\left(X_{\alpha}-X\right)
$$

it follows that $\phi\left(X_{\alpha}\right)$ converges to $\phi(X) . X^{*} X_{\alpha}$ and $X_{\alpha} X^{*}$ have the same limit so $\phi\left(X^{*} X\right)=0$. Hence $I$ is a left ultra-weakly closed ideal. So there exists a unique projection $E$ in $M$ such that $I=\{T / T E=T\}$. $U T U^{-1} \in I$ for all $U \in U(N)$ by stability. So $U E U^{-1}=E$. So $E \in N^{c}$. So $E=\phi(E)=0$. So if $\phi\left(X^{*} X\right)=0$ then $X=X E=0$, so $\phi$ is faithful.

Now let $G$ be a subgroup of $U(M)$. Let $N$ be the von Neumann algebra generated by $G$.

Lemma 3. A Schwartz map relative to $(G, M)$ is an expectation onto $N^{c}$.

Proof. Let $P$ be the Schwartz map. As $P(X)$ commutes with all unitaries of $G$, $P(X)$ is in $N^{c}$. Now if $A$ is in $N^{c}, C_{G}[A]$ reduces to the element $A$. So $P(A)=A$. So $P^{2}=P$. $N^{c}$ is hence the range of $P$ and $P(I)=I$. Now to show that $\|P\|<1$. Let $T=\sum_{i=1}^{n} \alpha_{i} U_{i} A U_{i}^{-1}$ where $\alpha_{i}>0$ and $\sum \alpha_{i}=1$. Then $\|T\|<\|A\|$. Because $P(A)$ is in $C_{G}[A]$ this means that there exists a net $T_{\alpha}$ of the same form as $T$ such that $T_{\alpha}$ converges strongly to $P(A)$. Let $X$ be a vector of norm one. $\left\|T_{\alpha} X\right\|$ converges to $\|P(A) X\|$ but $\left\|T_{\alpha} X\right\|<\|A\|$. So $\|P(A)\|<\|A\|$. By a result of J. Tomiyama [7], this implies that $P$ is an expectation.

Lemma 4. If $G$ is amenable, $S(G, M)$ is nonvoid.

Proof. Let $\lambda$ be a mean. Let $\xi$ and $\eta$ be 2 vectors. Considering $U$ as the variable, $\lambda\left(U^{-1} X U \xi, \eta\right)$ is a bounded hermitian form. By the Riez Lemma there exists an operator $E_{\lambda}$ such that $\lambda\left(U^{-1} X U \xi, \eta\right)=\left(E_{\lambda}(X) \xi, \eta\right)$. It was shown in [1] that $E_{\lambda}$ is in $S(G, M)$.

LEMMA 5. Let $M$ be finite and countably decomposable, let $G$ be any subgroup of $U(N)$, then $S(G, M)$ is nonvoid. (In particular if $N$ is any von Neumann subalgebra of $M$, then $S(U(N), M)$ is nonvoid.)

Proof. Let $\operatorname{Tr}$ be a faithful, normal, finite trace on $M$ [3]. By finiteness there exists a faithful, normal expectation $\phi$ of $M$ on $N^{c}$ such that $\operatorname{Tr}(X B)=\operatorname{Tr}(\phi(X) B)$ for all $X$ in $M$ and all $B$ in $N^{c}$. Hence $\phi\left(V X V^{-1}\right)=\phi(X)$ for all $X$ in $M$ and all $V$ in $U\left(N^{c c}\right) \supset U(N)$. Now to show $\phi(X)$ is in $C_{G}[X] . C_{G}[X]$ intersects $N^{c}$ [3]. Let $T$ be in $C_{G}[X] \cap N^{c}$, then by normality $T=\phi(T)=\phi(X)$. Hence $\phi$ is in $S(G, M)$.

Let $G$ be a subgroup of $U(M)$. Let $N$ be the von Neumann algebra generated by $G$. 
LEMMA 6. If $S(G, M)$ contains a normal map $\phi$, then $S(G, M)$ reduces to $\phi$, and so does $S(U(N), M)$. Moreover $C_{G}[X]$ intersects $N^{c}$ in just one point.

Proof. Let $T$ be in $C_{G}[X]$, by normality $\phi(T)=\phi(X)$. Now let $T$ be in $C_{G}[X] \cap N^{c}$. Then $T=\phi(T)$ by Lemma 3. So $T$ is the unique point in $C_{G}[X] \cap N^{c}$. By normality $\phi$ is $U(N)$ stable, so $S(U(N), M)=\{\phi\}$.

Lemma 7. Let $\operatorname{Tr}$ be a faithful, normal, semifinite trace on $M$. Let $G$ be a subgroup of $U(M)$ and $N$ the von Neumann algebra generated by $G$. Suppose $S(G, M)$ is sufficient, then the restriction of $\operatorname{Tr}$ to $N^{c}$ is semifinite.

Proof. In this proof the notation of [3] will be used. Let $\mathscr{M}$ be the ideal whose positive part consists of positive operators $A$ such that $\operatorname{Tr} A<\infty$. Consider $\mathscr{M}^{1 / 2}$. If $A$ is in $\mathscr{M}^{1 / 2}, C_{G}[A] \subset \mathscr{M}^{1 / 2}$ and $C_{G}[A] \cap N^{c}$ is nonvoid [3]. Let $S$ be a positive operator in $N^{c}, S \neq 0$. To show that there exists $S_{1} \neq 0, S_{1} \leqq S$ where $S_{1}$ is a positive operator of $N^{c} \cap M$. Let $A$ be in $\mathscr{M}$ such that $0 \leqq A \leqq I$. Let $P_{\alpha}$ be in $S(G, M)$. Then $S \geqq \sqrt{ } S P_{\alpha}(A) \sqrt{ } S=P_{\alpha}(\sqrt{ } S A \sqrt{ } S)$. $A$ can be picked such that $\sqrt{ } S A \sqrt{ } S \neq 0$ or else $A \sqrt{ } S=0$ for all $A$ positive in $\mathscr{M}$. By semifiniteness there would exist a net $A_{\alpha}$ converging weakly to $I$ so $I \sqrt{ } S=0$. So $S=0$, a contradiction. Pick $A$ then so that $\sqrt{ } S A \sqrt{ } S \neq 0$. Let $H=\sqrt{ } S A \sqrt{ } S$ then $H$ is in $\mathscr{M}^{1 / 2} . P_{\alpha}(\sqrt{ } H)$ is in $\mathscr{M}^{1 / 2} \cap N^{c}$. So $\left[P_{\alpha}(\sqrt{ } H)\right]^{2}$ is in $\mathscr{M} \cap N^{c}$. So $\left(P_{\alpha}(\sqrt{ } H)\right)^{2} \leqq P_{\alpha}(H) \leqq S$. By sufficiency, there exists an $\alpha_{0}$ such that $P_{\alpha_{0}}(\sqrt{ } H) \neq 0$. Choose $S_{1}=\left(P_{\alpha_{0}}(\sqrt{ } H)\right)^{2}$.

THEOREM 1. Let $\operatorname{Tr}$ be a faithful, semifinite trace of $M$. Let $N$ be a von Neumann subalgebra of $M$ and assume that the restriction of $\operatorname{Tr}$ to $N$ is semifinite, then there exists a normal, faithful $U\left(N^{c}\right)$-stable expectation $\phi$ of $M$ on $N$ such that $\operatorname{Tr}(A \phi(X))$ $=\operatorname{Tr}(A X)$ for all $X$ in $M$ and all $A$ in $N$ such that $\operatorname{Tr}|A|<\infty$.

Proof. Using the notations of the above lemma, let $A$ and $B$ be in $\mathscr{M}^{1 / 2} \cap N$ (that intersection is nonvoid), define $(A, B)=\operatorname{Tr}\left(A B^{*}\right)$. Choose $X$ positive in $M$ and define $[A, B]=\operatorname{Tr}\left(A B^{*} X\right)$. [ , ] is a bounded hermitian form respectively to $($,$) . Let k$ be the completion of $\mathscr{M}^{1 / 2}$ under ( , ). By the Riez Lemma there exists an operator $\phi(X)$ in $L(k)$ such that $[A, B]=(\phi(X)(A), B)$. Now: Let $R_{c}$ denote the right multiplication by $C$, where $C$ is in $\mathscr{M}^{1 / 2}$.

$$
\begin{aligned}
& \left(R_{c} \phi(X)(A), B\right)=\left(\phi(X)(A), B C^{*}\right)=\left[A, B C^{*}\right]=\operatorname{Tr}\left(A C B^{*} X\right), \\
& \left(\phi(X) R_{c}(A), B\right)=\left[R_{c}(A), B\right]=[A C, B]=\operatorname{Tr}\left(A C B^{*} X\right)
\end{aligned}
$$

so $R_{c} \phi(X)=\phi(X) R_{c}$.

By the commutation theorem [3] this implies that $\phi(X)(A)$ is a left multiplication by an element of $N$. Call that element $\phi(X)$. Then $\operatorname{Tr}\left(A B^{*} X\right)=\operatorname{Tr}\left(\phi(X) A B^{*}\right)$ $=\operatorname{Tr}\left(A B^{*} \phi(X)\right)$ for all $A$ and $B$ in $\mathscr{M}^{1 / 2} \cap N$ and all $X$ positive in $M . \phi$ can then be extended in the obvious fashion to all of $M$. As $\operatorname{Tr}$ is faithful, normal, it is easy 
to see that $\phi$ is faithful, normal, and $U\left(N^{c}\right)$ stable. For example to check that $\phi$ is $U\left(N^{c}\right)$ stable; let $V$ be in $U\left(N^{c}\right)$, let $A$ be in $N$, then:

$$
\operatorname{Tr}\left(A \phi\left(V X V^{-1}\right)\right)=\operatorname{Tr}\left(A V X V^{-1}\right)=\operatorname{Tr}\left(V A X V^{-1}\right)=\operatorname{Tr}(A X)=\operatorname{Tr}(A \phi(X)) .
$$

So $\left.\operatorname{Tr}\left[A\left(\phi V X V^{-1}\right) \cdot \phi(X)\right)\right]=0$ for all $A$ in $N \cap \mathscr{M}$. Since $\operatorname{Tr}$ is semifinite on $N$, let $P_{\alpha}$ be a family of orthogonal projections of $N$ such that $\operatorname{Tr} P_{\alpha}<\infty$ and $\sum P_{\alpha}=I$. Make $A=\left(\phi\left(V X V^{-1}\right)-\phi(X)\right) * P_{\alpha}$. One has $P_{\alpha}\left(\phi\left(V X V^{-1}\right)-\phi(X)\right)=0$ for all $\alpha$, i.e. $\phi\left(V X V^{-1}\right)=\phi(X)$.

THeOREM 2. Suppose $M$ has a faithful, normal, semifinite trace Tr. Suppose $S(G, M)$ is sufficient, then there exists a faithful, normal $U\left(N^{c c}\right)$ stable expectation of $M$ on $N^{c}$. ( $N$ is the algebra generated by $G$.)

Proof. By Lemma 7 the restriction of $\operatorname{Tr}$ to $N^{c}$ is semifinite. By Theorem 1 there exists a normal, faithful, $U\left(N^{c c}\right)$ stable expectation $\phi$ of $M$ on $N^{c}$ such that $\operatorname{Tr}(A X)$ $=\operatorname{Tr}(A \phi(X))$ for all $A$ in $N^{c}$ such that $\operatorname{Tr}|A|<\infty$. Now $\phi$ is in $S(G, M)$. Indeed $\phi$ is $G$-stable and if $P$ is in $S(G, M)$ then $\phi(P(X))=P(X)$ (as $\phi$ is the identity on $N^{c}$ ). By normality $\phi(P(X))=\phi(X)$. So $P=\phi$. Hence $\phi$ is a normal, faithful, $U\left(N^{c c}\right)$ stable expectation of $M$ on $N^{c}$ by Lemma 3.

The above theorem says that if there is a sufficient number of $G$-stable expectations of $M$ on $N^{c}$, there is a faithful, normal one which in fact is more than $G$-stable it is $U\left(N^{c c}\right)$ stable.

COROLlary 1. With the above hypothesis $N^{c c}$ is finite.

Proof. By the above theorem $S\left(U\left(N^{c c}\right), M\right)$ is nonvoid. Let $P$ be in $S\left(U\left(N^{c c}\right), M\right)$. Let $A$ be in $N^{c c}$, let $C(A)$ be the norm closure of the convex hull $K_{A}$ of points of the form $U A U^{-1}$ as $U$ ranges over $U\left(N^{c c}\right)$. Consider $C(A) \cap Z$ where $Z$ is the center of $N^{c c}$. $C(A) \cap Z$ is nonvoid [3]. By [3] it is sufficient to show that $C(A) \cap Z$ reduces to one point. $P$ is constant on $K_{A}$ hence on $C(A)$. Let $T_{1}$ and $T_{2}$ be in $C(A) \cap Z$, then $T_{1}=P\left(T_{1}\right)=P\left(T_{2}\right)=T_{2}$, so $N^{c c}$ is finite. In particular $N$ is finite.

COROLlary 2. With the above hypothesis $N^{c}$ can not be purely infinite.

Proof. In [7] J. Tomiyama proved that if $\pi$ is an expectation from a semifinite algebra $M$ onto a purely infinite subalgebra $\mathscr{A}$, then $\pi$ is always singular, i.e. $\pi$ is not normal. Since there exists a normal expectation from $M$ on $N^{c}, N^{c}$ is not purely infinite.

COROllary 3. With the above hypothesis if $M$ is of type $\mathrm{I}$, so is $N^{c}$.

Proof. In [7] it has been shown that if there exists an expectation from $M$ of type I to a subalgebra of type II, that expectation is not normal. By the above corollary $N^{c}$ has no part of type III and hence no part II or III are present, so $N^{c}$ is of type $\mathrm{I}$.

Let $G$ be a subgroup of $U(M)$. Let $N$ be generated by $G$.

COROLlaRY 4. Let $M$ be a countably decomposable von Neumann algebra and consider the following conditions: 
(1) $N$ is finite and there exists a faithful, normal expectation $\phi$ of $M$ and $N$.

(2) There exists a faithful, normal state $\rho$ of $M$ such that $\rho\left(U X U^{-1}\right)=\rho(X)$ for all $U$ in $G$.

(3) There exists a faithful, normal expectation $\psi$ of $M$ on $N^{c}$ such that $\psi\left(V X V^{-1}\right)$ $=\psi(X)$ for all $V$ in $U(N)$.

(4) $S(G, M)$ is sufficient and $M$ has a faithful, semifinite normal trace $\operatorname{Tr}$.

Then (1) and (2) are equivalent. If $S(G, M)$ is nonvoid, (2) and (3) are equivalent. Finally (4) always implies (3).

Proof. Assume (1), then there exists a faithful, normal finite trace $\lambda$ on $N$. Let $r(X)=\lambda[\phi(X)]$. Clearly $r$ is faithful, normal and bounded. Let $U$ be in $G$, then $r\left(U X U^{-1}\right)=\lambda \phi\left(U X U^{-1}\right)=\lambda \phi(X)=r(X)$. Normalizing $r,(2)$ is established.

Assume (2). By a classical Hilbert algebra argument one can show that there exists an expectation $\phi$ such that $\rho(A X)=\rho(A \phi(X))$ for all $A$ in $N$ and all $X$ in $M$. $\phi$ will satisfy (1).

Assume now (2) together with the fact that $S(G, M)$ is nonvoid. Let $P$ be in $S(G, M) . \rho$ is constant on $C_{G}[A]$. Hence $\rho(A)=\rho(P(A))$. This shows that $P$ is faithful, normal and satisfies $P\left(V A V^{-1}\right)=P(A)$, for all $V$ in $U(N)$. For example to check that $P(A)=P\left(V A V^{-1}\right)$ :

Let $B$ be any element of $N^{c}$.

$$
\begin{aligned}
& \rho\left(B V A V^{-1}\right)=\rho\left(P\left(B V A V^{-1}\right)\right)=\rho\left(B P\left(V A V^{-1}\right)\right), \\
& \rho\left(B V A V^{-1}\right)=\rho\left(V B A V^{-1}\right)=\rho(B A)=\rho(B P(A)) .
\end{aligned}
$$

Choose $B=\left(P\left(V A V^{-1}\right)-P(A)\right)^{*}$, by faithfulness of $\rho, P\left(V A V^{-1}\right)=P(A)$.

Assume now (3). By countable decomposability there exists a faithful, normal state $\sigma$ of $M$ (get a maximal set of orthogonal projections $P_{n}$ of $M$ where each $P_{n}$ is the projection on $\left[M^{\prime} x_{n}\right]$, and let $\sigma=\sum W_{x_{n}}$ (notation of [3]). Let $\rho(X)=\sigma \psi(X)$. $\rho$ in the state of (2).

Finally to show that (4) implies (3). By Theorem 2 there exists a faithful, normal expectation of $M$ on $N^{c}$, call it $\Psi$ such that $\operatorname{Tr}(X A)=\operatorname{Tr}(\Psi(X) A)$ for all $A$ in $\mathscr{M} N^{c}$. As above one shows that $\Psi\left(V X V^{-1}\right)=\Psi(X)$.

COROllary 5. If $S(G, L(h))$ is sufficient, $N$, the algebra generated by $G$, is atomic.

Proof. By Theorem 2 there exists a faithful, normal, expectation of $L(h)$ on $N^{\prime}$ which is $U(N)$ stable. By Corollary $3, N^{\prime}$ is of Type I, hence so is $N$ [3]. Also $N$ is finite by Corollary 1. Let $Z$ be the center of $N$. Any projection of $N$ dominates an abelian projection in $N$, call it $P \neq 0$. If $Q$ is a projection of $N$ such that $Q \leqq P$, then $Q=P C$ where $C$ is a projection of $Z$. Since $Z$ is atomic [3], $Q$ and hence $P$ dominates a minimal projection. So $N$ is atomic.

REMARKS. The following statements are trivial to see:

(1) If $S(G, L(h))$ is sufficient then there exists a normal expectation $\phi$ from $L(h)$ to $N^{\prime}$ such that $\phi\left(U X U^{-1}\right)=\phi(X)$ for all $U$ in $G$, this is part of Corollary 4 .

(2) Assuming $S(G, L(h))$ contains a normal map, then $S(G, L(h))$ is sufficient. 
Let $\pi$ be a normal map, then $\pi$ is faithful. Indeed: by normality $\pi\left(U X U^{-1}\right)=\pi(X)$ for all $U$ in $U(N)$. Assume that $P$ is a projection such that $\pi(P)=0$. Let $Q=$ Sup $U P U^{-1}$ is $U \in U(N)$. Then $Q=V Q V^{-1}$ for all $V$ in $U(N)$, so $Q$ is in $N^{\prime}$. Hence $Q=\pi(Q)=0$. So $P=0$.

Definition. Two groups of unitaries are equivalent if they generate the same von Neumann algebra.

TheOREM 3. Assume $S(G, L(h))$ contains a normal map $\pi$, then $G$ is equivalent to a countable direct sum of finite groups.

Proof. By Lemma $6, S(G, L(h))=\{\pi\}$. By the above remark $\pi$ is faithful and by normality $\pi$ is in $S(U(N), L(h))$. By Corollary $1, N$ is finite. Let $Z$ be the center of $N$. By Corollary $5, Z$ is atomic. Pick a maximal set of orthogonal minimal projections, $C_{n}$ of $Z$ such that $N=\oplus N_{c_{n}} . N_{c_{n}}$ is a factor of Type $\mathrm{I}_{n} . N_{c_{n}}$ is isomorphic to $n \times n$ matrices, so $N_{c_{n}}$ is generated by a finite group $K_{n}$ of unitaries. Let $K=\oplus K_{n}$ (all components are the identity except a finite number). The algebra generated by $K$ contains all $N_{c_{n}}$, so it contains $N$. Each $K_{n}$ is a subgroup of $U(N)$. So the algebra generated by $K$ is $N$.

Let $M$ be a von Neuman algebra and let $G$ be a subgroup of $U(M)$.

Definition. $G$ will satisfy condition $(F)$ if

(1) There exists orthogonal projections $C_{\alpha}$ of $N^{\prime}$ ( $N$ is the algebra generated by $G)$ such that $I=\sum C_{\alpha}$ and $\left|G C_{\alpha}\right|<\infty$.

(2) For every $U$ in $G, U C_{\alpha}=C_{\alpha}$ for all but a finite number of $\alpha$.

THEOREM 4. If $G$ has property $(F)$, then $G$ is a countable direct sum of finite groups, and $S(G, M)$ is sufficient.

Proof. Define a map $\pi_{\alpha}$ on $G$ by $\pi_{\alpha}(U)=U C_{\alpha} \cdot \pi_{\alpha}$ is clearly a homomorphism of $G$ and $\pi_{\alpha}(G)$ is finite. Also the intersection of all kernels of $\pi_{\alpha}$ is $I$. Let $F_{\alpha}=\pi_{\alpha}(G)$, then by definition of condition $(F), G=\oplus F_{\alpha}$. As each $F_{\alpha}$ is finite $G$ is amenable since it is locally finite. So $S(G, M)$ is nonvoid by Lemma 4 . Now let $A$ be a positive operator in $M$, let $P$ be in $S(G, M)$ and suppose $P(A)=0$ for all $P$ in $S(G, M)$. If $A \neq 0, C_{\alpha} A C_{\alpha} \neq 0$ for some $C_{\alpha}$, call $\alpha_{0}$ such an $\alpha . C_{\alpha_{0}} P(A) C_{\alpha_{0}}=$ $P\left(C_{\alpha_{0}} A C_{\alpha_{0}}\right) \in C_{G}\left[C_{\alpha_{0}} A C_{\alpha_{0}}\right]$. Let $H$ be all elements of $G$ where the $\alpha_{0}$ component is the identity. Then $G=H F_{\alpha_{0}}$. Let $U$ be in $G$, then $U$ is uniquely written as $U=V W$ where $V$ is in $H$ and $W$ in $F_{\alpha_{0}} . U C_{\alpha_{0}} A C_{\alpha_{0}} U^{-1}=W C_{\alpha_{0}} A C_{\alpha_{0}} W^{-1}$ but there is only a finite number of $U C_{\alpha_{0}} A C_{\alpha_{0}} U^{-1}$. Hence $C_{G}\left[C_{\alpha_{0}} A C_{\alpha_{0}}\right]$ is the convex hull of $W C_{\alpha_{0}} A C_{\alpha_{0}} W^{-1}$ as $W$ ranges in $F_{\alpha_{0}}$. So $0=P\left(C_{\alpha_{0}} A C_{\alpha_{0}}\right)=\sum_{i=1}^{n} \alpha_{i} W_{i} C_{\alpha_{0}} A C_{\alpha_{0}} W_{i}^{-1}$. So $C_{\alpha_{0}} A C_{\alpha_{0}}=0$, a contradiction. So $A=0$ and $S(G, M)$ is sufficient.

REMARK. While proving Theorem 3 it has been shown that if $N$ is a finite atomic von Neumann algebra, then $N$ is generated by a direct sum of finite groups $K_{n}$.

4. Uniqueness properties.

LEMma 8. If there exists only one faithful, normal expectation $\Phi$ of $M$ on $N$ then $N^{c} \subset N$. 
Proof. Let $\varepsilon>0$. Let $H$ be a positive operator in $N^{c}$ such that $H>\varepsilon I>0, \Phi(H)$ is in $N, \Phi(H)>\varepsilon I$. Let $X$ be in $N$, then $X \Phi(H)=\Phi(X H)=\Phi(H) X$. So $\Phi(H)$ is in $N^{\prime}$, so in $N \cap N^{\prime}=Z_{N}$. Define $\pi(X)=\Phi(H)^{-1} \Phi\left(H^{1 / 2} X H^{1 / 2}\right)$.

Clearly $\pi$ is another expectation of $M$ on $N$, by uniqueness $\pi=\Phi$. So $\Phi\left(H^{1 / 2} X H^{1 / 2}\right)$ $=\Phi(H) \Phi(X)$, in particular if $X=H$ then $\Phi(H)^{2}=\Phi(H)^{2}$; this holds for any selfadjoint operator in $N^{c}$ which is positive. Let $H$ be any selfadjoint operator in $N^{c}$. Pick $C>0$ such that $C I+H>\varepsilon I$ then $[\Phi(C I+H)]^{2}=\Phi\left[(C I+H)^{2}\right]$ so $\Phi(H)^{2}=$ $\Phi(H)^{2}$. Let $P$ be a projection in $N^{c}$, then $(P-\Phi(P))^{2}>0$ so $\Phi(P-\Phi(P))^{2}=$ $(\Phi(P)-\Phi(P))^{2}=0$. By faithfulness $P=\Phi(P)$ i.e. $P$ is in $N$ so $N^{c} \subset N$.

Lemma 9. Let $N$ be normal in $M$ (i.e. $N^{c c}=N$ ) if a faithful expectation exists from $M$ to $N$ then $N^{c} \subset N$. If $N^{c} \subset N$ and if a normal expectation $\Phi$ exists from $M$ to $N$ then $\Phi$ is the only such normal expectation.

Proof. The first part was shown in Lemma 1. Now to show the second part: As $N^{c} \subset N, N^{c}$ is the center of $N$ in particular $N^{c}$ is abelian. Hence, $U\left(N^{c}\right)$ is amenable, so $S\left(U\left(N^{c}\right), M\right)$ is nonvoid by Lemma 4. Let $P$ be in $S\left(U\left(N^{c}\right), M\right)$, then $P$ is an expectation on $N^{c c} \cap M$ by Lemma 3. So $P$ is an expectation on $N$. Let $\Phi(P(X))=\Phi(X)$. Also $\Phi(P(X))=P(X)$, so $\Phi=P$. This shows that if $\Phi$ exists, it is unique.

THEOREM 5. Assume the following conditions:

(1) $N^{c} \subset N$,

(2) $N$ is finite,

(3) $M$ is semifinite.

Then there exists at most one normal expectation $\phi$.

Proof. $N^{c}$ is the center of $N$, by finiteness the map (notation of [3]) is defined from $N$ to $N^{c}$. If $X$ is in $M$, define $\Psi(X)=(\phi(X))^{\natural}$. $\Psi$ is a normal map and $S\left(U\left(N^{c}\right), M\right)$ is nonvoid. Let $P$ be in $S\left(U\left(N^{c}\right), M\right)$. If $X$ is in $\mathscr{M}^{1 / 2}, C_{U(N)}[X]$ $\cap \mathscr{M}^{1 / 2}$ and $C_{U(N)}[X]$ intersect $N^{c}$. Let $T$ be in $C_{U(N)}[X] \cap N^{c} . \Psi$ is invariant under $U(N)$, so $T=\Psi(T)=\Psi(X)$. So $N^{c} \cap C_{U(N)}[X]=\{\Psi(X)\}$. If $\phi_{1}$ is another normal expectation of $M$ on $N$, then define $\Psi_{1}(X)=\left[\phi_{1}(X)\right]^{\natural}$. Also $N^{c} \cap C_{U(N)}[X]=$ $\left\{\Psi_{1}(X)\right\}$, so $\Psi=\Psi_{1}$ on $\mathscr{M}^{1 / 2}$, hence on $M$.

Let $\lambda$ be any normal, finite trace on $N$. Then: $\lambda \phi(X)=\lambda \Psi(X)=\lambda \Psi_{1}(X)=\lambda \phi_{1}(X)$. Since the $\lambda$ form a complete set $\phi=\phi_{1}$.

In conclusion consider the following problem. Let $N$ be a von Neumann algebra. Suppose there exists sufficiently many expectations of $M$ on $N$. Is $N$ relatively semifinite? An answer to that problem was given when the expectations are of a certain type (Lemma 7).

\section{BIBLIOGRAPHY}

1. W. B. Arveson, Analyticity in operator algebras, Amer. J. Math. 89 (1967), 578-642. MR 36 \#6946.

2. J. Dixmier, Formes linéaires sur un anneau d'opérateurs, Bull. Soc. Math. France 81 (1953), 9-39. MR 15, 539. 
3. J. Dixmier, Les algèbres d'opérateurs dans l'espace hilbertien, Gauthier-Villars, Paris, 1957. MR 20 \#1234.

4. E. Følner, On groups with Full Banach mean value, Math. Scand. 3 (1955), 243-254. MR 18, 51.

5. I. Namioka, Folner's conditions for amenable semi-groups, Math. Scand. 15 (1964), 18-28. MR 31 \#5062.

6. J. Schwartz, Two finite, non-hyperfinite, non-isomorphic factors, Comm. Pure Appl. Math. 16 (1963) 19-26. MR 26 \#6812.

7. J. Tomiyama, On the projection of norm one in $W^{*}$-algebras, Proc. Japan Acad. 33 (1957), 608-612. MR 20 \#2635.

8. - On the product projection of norm one in the direct product of operator algebras, Tôhoku Math. J. (2) 11 (1959), 305-313. MR 21 \#7453.

9. H. Umegaki, Conditional expectation in an operator algebra, Tôhoku Math. J. (2) 6 (1954), 177-181. MR 16, 936.

INDIANA STATE UNIVERSITY,

TerRe HaUte, INDIANA 